\title{
Spherically Symmetric Dirac Operators with Variable Mass and Potentials Infinite at Infinity
}

\author{
By
}

Karl Michael SchmidT* and Osanobu YAMADA**

\begin{abstract}
We study the spectrum of spherically symmetric Dirac operators in three-dimensional space with potentials tending to infinity at infinity under weak regularity assumptions. We prove that purely absolutely continuous spectrum covers the whole real line if the potential dominates the mass, or scalar potential, term. In the situation where the potential and the scalar potential are identical, the positive part of the spectrum is purely discrete: we show that the negative half-line is filled with purely absolutely continuous spectrum in this case.
\end{abstract}

\section{$\S 1$. Introduction}

In a recent paper [20] the spectral properties of the three-dimensional Dirac operator

$$
H=\alpha \cdot p+m(x) \beta+q(x) I_{4} \quad\left(x \in \mathbb{R}^{3}\right)
$$

(where $p=-i \nabla, i^{2}=-1, I_{n}$ is the $n \times n$ unit matrix, and $\alpha_{0}=\beta, \alpha_{1}, \alpha_{2}, \alpha_{3}$ are Hermitian $4 \times 4$ matrices satisfying the anti-commutation relations

$$
\left.\alpha_{j} \alpha_{k}+\alpha_{k} \alpha_{j}=2 \delta_{j k} \quad(j, k \in\{0,1,2,3\})\right)
$$

were studied under the condition that the real-valued coefficient function $m$ tends to $\infty$ (or $-\infty$ ) as $|x| \rightarrow \infty$. For constant $m, H$ is the Hamilton operator describing a relativistic quantum mechanical particle of mass $m$ moving in an external force field of (real-valued) potential $q$. As a non-constant function, $m$ can also take the role of a so-called scalar potential, which has been discussed in the physical literature as a model of quark confinement (cf. the references in [20], Thaller [14] p. 305).

Communicated by T. Kawai, June 2, 1997.

1991 Mathematics Subject Classfication(s): 81Q10, 35Q40, 34L40

* Mathematisches Institut der Universitat, Theresienstr. 39, D-80333 Munchen, Germany.

**Department of Mathematics, Ritsumeikan University, Shiga 525-8577, Japan. 
In [20] it is shown that if $m$ dominates $q$ and tends to infinity as $|x| \rightarrow \infty$, then the spectrum of the Dirac operator $H$ is purely discrete; if $m$ coincides identically with $q$, and tends to infinity (in addition to certain regularity requirements), then the positive part of the spectrum of $H$ is purely discrete. Furthermore, if $m \equiv q$ is of at most quadratic growth, the negative half-line is filled with purely continuous spectrum of $H$. It seems to be a rather more delicate question to determine the quality (absolute continuity or otherwise) of the continuous part of the spectrum of $H$ in the case $m \equiv q$, or even the overall structure of the spectrum in the situation where $q$ dominates $m$ and tends to infinity. It is the purpose of the present paper to address this question under the additional assumption that $m$ and $q$ are spherically symmetric functions; then the operator $H$ is spherically symmetric in the sense that rotations in space lead to unitarily equivalent operators.

By the well-known procedure of separation in spherical polar coordinates (cf. Weidmann [18] Appendix to Section 1), $H$ is then unitarily equivalent to the direct sum of the countable family of one-dimensional Dirac operators on the half-line $r>0$,

$$
h_{k}=\sigma_{2} p+\frac{k}{r} \sigma_{1}+m(r) \sigma_{3}+q(r) I_{2} \quad(k \in \mathbb{Z} \backslash\{0\}) .
$$

where $p=-i \frac{d}{d r}$, and $\sigma_{1}=\left(\begin{array}{ll}0 & 1 \\ 1 & 0\end{array}\right), \sigma_{2}=\left(\begin{array}{cc}0 & -i \\ i & 0\end{array}\right), \sigma_{3}=\left(\begin{array}{cc}1 & 0 \\ 0 & -1\end{array}\right)$ are the Pauli matrices. If $m$ and $q$ are regular, i.e. locally integrable on $(0, \infty)$ and integrable at 0 , this Dirac system is in the limit point case at 0 for all $k \in \mathbb{Z} \backslash\{0\}$ by virtue of the singular angular momentum term $k \sigma_{1} / r([11]$ Lemma 1$)$; moreover, it is in the limit point case at $\infty$ as well (Weidmann [18] Corollary to Theorem 6.8), and consequently the minimal operator associated with the formal expression $h_{k}$ is essentially self-adjoint by the Weyl theory (Weidmann [18] Theorem 5.8). We denote the unique self-adjoint realization again by $h_{k} ; H$ : $=\bigoplus_{k \in \mathbb{Z} \backslash\{0\}} h_{k}$ is a self-adjoint realization of the three-dimensional Dirac operator.

The study of the spectrum of $H$ can then essentially be reduced to that of the individual one-dimensional operators $h_{k}$. The spectral properties of half-line Dirac operators with potentials which do not approach a finite limit at infinity, have been studied previously in the literature in different special situations. For example, Hinton and Shaw [8], extending the work of Roos and Sangren [9], give conditions for a Dirac system with dominant $m$ to have purely discrete spectrum. On the other hand, Evans and Harris [4] derive lower bounds on the absolute value of eigenvalues in a situation with dominant $q$. In the case of the electron Dirac operator $(m=$ const). Erdélyi [3] has established that the spectrum of $h_{k}$ is purely absolutely continuous and covers the whole real line provided $q$ is locally absolutely continuous, 


$$
\lim _{r \rightarrow \infty} q(r)=\infty, \text { and } \int \frac{\left|q^{\prime}\right|}{q^{2}}<\infty,
$$

thus refining a result of Titchmarsh [15] that had been anticipated, in a formal way, by Rose and Newton [10]. Recently a different proof of this assertion (for $m \equiv 1$ ), based on the Gilbert-Pearson method, was given by [12] under the slightly weaker hypotheses $q \in B V_{\text {loc }}(0, \infty)$,

$$
\lim _{r \rightarrow \infty} q(r)=\infty, \text { and } \frac{1}{q} \in B V(\cdot, \infty) .
$$

(Here $B V(I)$ denotes the space of functions of bounded variation on the interval $I \subset \mathbb{R}$, and $B V_{\text {loc }}(I)$ is the corresponding local space. Generally, if $X(I)$ is a space of functions on the real interval $I$, we here and below use the notation

$$
X(\cdot, \infty):=\{f \mid \text { there is } a \in \mathbb{R} \text { such that } f \in X([a, \infty))\} \text {.) }
$$

In this paper we consider, following the methods of [12], the case of non-constant $m$; this also requires that the angular momentum term be handled in a different way than the perturbative treatment indicated in [12], (cf. Remark 6 below). We prove that (under certain weak regularity assumptions) the negative part of the spectrum of $H$ is purely absolutely continuous if

$$
m(r) \equiv q(r) \rightarrow \infty \quad(r \rightarrow \infty),
$$

and that the whole real line is filled with purely absolutely continuous spectrum of $H$ if

$$
m(r) \ll q(r) \rightarrow \infty \quad(r \rightarrow \infty)
$$

\section{$\S 2 . \quad$ Results}

We prove the following theorem in Section 3.

Theorem 1. Let $q \in L_{\mathrm{loc}}^{1}([0, \infty)), m \in A C_{\mathrm{loc}}([0, \infty))$, and assume that

(A1) $\lim _{r \rightarrow \infty} q(r)=\infty$,

(A2) $\quad \liminf _{r \rightarrow \infty}|m(r)|>0 . \quad \lim \sup _{r \rightarrow \infty}\left|\frac{m(r)}{q(r)}\right|<1$,

(A3) $\frac{m}{q-\lambda} \in B V(\cdot, \infty) \quad(\forall \lambda \in \mathbb{R})$.

(A4) $\frac{m^{\prime}}{r m q} \in L^{1}(\cdot, \infty)$.

Then $\sigma_{a c}(H)=\mathbb{R}$, and $\sigma_{s}(H)=\varnothing$.

Remark 1. By unitary equivalence, the same result holds true if, instead of 
(A1), we assume $\lim _{r \rightarrow \infty} q(r)=-\infty$. In view of the condition (A3) we may rewrite the latter condition in (A2) as follows;

$$
\lim _{r \rightarrow \infty}\left|\frac{m(r)}{q(r)}\right|<1
$$

Remark 2. If $m$ is constant, (A3) is true for all $\lambda \in \mathbb{R}$ if it is true for one real value of $\lambda$ (cf. Remark 8 in the Appendix). This does not hold in the general case ; for example, choosing $m(r):=2+\sin r, q(r)=r^{1 / 4} m(r)(r \in$ $[0, \infty)$ ), we find that (A1), (A2), (A4) and (A3) for $\lambda=0$ are satisfied, but (A3) for $\lambda \neq 0$ is not.

However, Proposition 5 (in the Appendix) shows that it is generally sufficient to assume (A3) for two distinct values of $\lambda$. Alternatively, if we assume, in . addition to (A1), that $q \in A C_{\mathrm{loc}}\left({ }^{\circ}, \infty\right)$ and $m q^{\prime} q^{-3} \in L^{1}(\cdot, \infty)$, then $m / q \in$ $B V(\circ, \infty)$ implies $m /(q-\lambda) \in B V(\circ, \infty)$ for all $\lambda \in \mathbb{R}$, by a straightforward application of Proposition 3 in the Appendix to $\frac{m}{q-\lambda}=\frac{q}{q-\lambda} \cdot \frac{m}{q}$.

Theorem 1 (cf. also Theorem 2 below), as well as the results of [12], Weidmann [16] $\S 8$, and Weidmann [17], strongly suggest that a condition of bounded variation of the coefficient functions is a natural setting for statements on absolute continuity of the spectrum of one-dimensional Hamiltonians. Nevertheless, assuming higher regularity of the coefficients (as will usually be given in concrete applications) one can reformulate the conditions of bounded variation in terms of integrability of derivatives, yielding hypotheses which may be more convenient to verify than (A3) itself. Thus we note

Corollary 1. Let $q, m \in A C_{\text {loc }}([0, \infty))$ and assume (A1), (A2), and

$$
\frac{m^{\prime}}{q}, \frac{m q^{\prime}}{q^{2}} \in L^{1}(\cdot, \infty) .
$$

Then $\sigma_{a c}(H)=\mathbb{R}, \sigma_{s}(H)=\emptyset$.

Proof. The assumption gives $\left(\frac{m}{q-\lambda}\right)^{\prime}=\frac{m^{\prime} q-q^{\prime} m-\lambda m^{\prime}}{(q-\lambda)^{2}} \in L^{1}(\cdot, \infty)$ for each $\lambda \in \mathbb{R}$, which implies (A3). Furthermore, by (A2) we have $r|m(r)| \rightarrow \infty(r \rightarrow \infty)$, and thus

$$
\frac{m^{\prime}}{r m q}=\frac{1}{r m} \cdot \frac{m^{\prime}}{q} \in L^{1}(\cdot, \infty)
$$

As a borderline case between the two situations in which either $q$ dominates $m$ (giving rise to purely absolutely continuous spectrum as shown above), or $m$ dominates $q$ (with purely discrete spectrum as a consequence of 
[20]), we then study the case in which $m$ and $q$ coincide identically. In Section 4 we prove the following result.

Theorem 2. Let $m \equiv q \in A C_{\mathrm{loc}}([0, \infty))$, and assume

(B1) $\lim q(r)=\infty$,

(B2) $\frac{q^{\prime}}{q^{3 / 2}} \in B V(\cdot, \infty) \cap L^{2}(\cdot, \infty)$.

Then $\sigma_{a c}(H)=(-\infty, 0], \sigma_{s}(H) \cap(-\infty, 0)=\emptyset$.

For the convenience of the reader, we include a corollary in which assumption (B2), which involves bounded variation, is replaced by a condition of integrability of derivatives.

Corollary 2. Let $m \equiv q \in C^{2}([0, \infty))$, and assume (B1) and

$$
\text { (B2) }, \frac{q^{\prime \prime}}{q^{3 / 2}}, \frac{\left(q^{\prime}\right)^{2}}{q^{5 / 2}} \in L^{1}(\cdot, \infty) \text {. }
$$

Then $\sigma_{a c}(H)=(-\infty, 0], \sigma_{s}(H) \cap(-\infty, 0)=\emptyset$.

Proof. $\left(\frac{q^{\prime}}{q^{3 / 2}}\right)^{\prime}=\frac{q^{\prime \prime}}{q^{3 / 2}}-\frac{3\left(q^{\prime}\right)^{2}}{2 q^{5 / 2}} \in L^{1}(\cdot, \infty)$ and $\left(\frac{q^{\prime}}{q^{3 / 2}}\right)^{2}=\frac{1}{q^{1 / 2}} \cdot \frac{\left(q^{\prime}\right)^{2}}{q^{5 / 2}} \in$ $L^{1}(\cdot, \infty)$ imply $(B 2)$.

Remark 3. Under the hypotheses of Corollary 2. every solution $u$ of $h_{k} u=\lambda u$ is twice continuously differentiable, and satisfies the ordinary differential equation system

$$
u_{1}^{\prime}+\frac{k}{r} u_{1}=\lambda u_{2}, \quad-\imath_{1}^{\prime \prime}+2 \lambda q u_{1}+\frac{k(k+1)}{r^{2}} u_{1}=\lambda^{2} u_{1}
$$

Along the lines of Dunford and Schwartz [2] Theorem XIII. 6.20, one can then prove the existence of a fundamental system $u_{+}, u_{-}$with asymptotic behaviour

$$
u_{ \pm}(r)=\left(\begin{array}{c}
q(r)^{-1 / 4}\left(e^{ \pm 2} \int_{1}^{r} \sqrt{\lambda^{2}-2 \lambda q}+o(1)\right) \\
\mp i \sqrt{\frac{2}{-\lambda}} q(r)^{1 / 4}\left(e^{ \pm \int_{1}^{r} \sqrt{\lambda^{2}-2 \lambda q}}+o(1)\right)
\end{array}\right)
$$

as $r \rightarrow \infty$, which implies the non-existence of subordinate solutions for negative $\lambda$. In order to achieve weaker regularity requirements, our proof of Theorem 2 follows a different approach, remaining fully in the context of Dirac systems without recourse to associated second order differential equations. 


\section{§ 3. The Case $m \ll q$}

In this Section we prove Theorem 1. We proceed as follows. As indicated in the Introduction, the spherically symmetric Dirac operator $H$ is unitarily equivalent to the countable direct sum of half-line operators with angular momentum term:

therefore we have

$$
H \cong \underset{k \in \mathbb{Z} \backslash\{0\}}{\bigoplus} h_{k}
$$

$$
\sigma_{a c}(H)=\overline{\bigcup_{k \in \mathbb{Z} \backslash\{0\}} \sigma_{a c}\left(h_{k}\right)}, \quad \sigma_{s}(H)=\overline{\bigcup_{k \in \mathbb{Z} \backslash\{0\}} \sigma_{s}\left(h_{k}\right)}
$$

(cf. [11]), and hence it is sufficient to prove that for each nonzero integer $k$; $h_{k}$ has purely absolutely continuous spectrum throughout the real line.

By the following proposition, this can be reduced to showing that all solutions of the eigenvalue equation for $h_{k}$, for all real values of the spectral parameter, are bounded at infinity.

Proposition $\mathbb{1}$. Let $a \geq-\infty, l, m, q \in L_{\text {loc }}^{1}((a, \infty))$ be real-valued functions, and $I \in \mathbb{R}$ an open interval. Let $h$ be a self-adjoint realization of the Dirac system

$$
\sigma_{2} p+m \sigma_{3}+l \sigma_{1}+q I_{2}
$$

on $(a, \infty)$. If, for every $\lambda \in I$, every solution $u$ of

$$
\left(\sigma_{2} p+m \sigma_{3}+l \sigma_{1}+q I_{2}\right) u=\lambda u
$$

is an element of $L^{\infty}(\cdot, \infty)$, then we have $I \subset \sigma_{a c}(h), \sigma_{s}(h) \cap I=\emptyset$.

Remark 4. It is possible to give a direct proof for this special case of the Gilbert-Pearson method for Dirac systems (Behncke [1] Lemma 1) along the lines of Simon [13], cf. the Appendix of [12], bypassing the complications of the general theory. Note that only a condition for each separate value of $\lambda$ is imposed in the above Proposition; however, in our situation, one could also apply Weidmann's theorem [19] instead of Proposition 1, after showing that the bounds on the solutions are locally uniform in $\lambda$.

The heart of the matter is contained in Proposition 2, in which a sufficient condition for the boundedness of all solutions of a general Dirac system

$$
\left(\sigma_{2} p+M \sigma_{3}+L \sigma_{1}+Q I_{2}\right) u=0
$$

with $\lim _{r \rightarrow \infty} Q(r)=\infty$, is given.

After proving Proposition 2, we finish the Proof of Theorem 1 by showing that $Q:=q-\lambda, M:=m$ and $L:=k / r$ satisfy the hypotheses of Proposition 2 for all real $\lambda$ and all non-zero integers $k$. 
Proposition 2. Let $a \geq-\infty, Q, M, L \in L_{\mathrm{loc}}^{1}(a, \infty)$ be real-valued functions such that

(C1) $\lim _{r \rightarrow \infty} Q(r)=\infty$,

(C2) $\lim _{r \rightarrow \infty} \sup \frac{W(r)}{Q(r)}<1$,

(C3) $\frac{W}{Q-W}, \frac{M}{Q-W}, \frac{L}{Q-W} \in B V(\cdot, \infty)$.

where $W:=\sqrt{M^{2}+L^{2}}$. Then every solution of $(*)$ is bounded at infinity. If $M \equiv 0[L \equiv 0]$, condition (C3) can be replaced by

$$
\text { (C3) } \frac{L}{Q-L} \in B V(\cdot, \infty) \quad\left[\frac{M}{Q-M} \in B V(\cdot, \infty)\right] \text {. }
$$

Corollary 3. Under the hypotheses of Proposition 2, let $r_{0}>a$. Then there is a constant $C>0$ such that

$$
\frac{\left|y\left(r_{0}\right)\right|^{2}}{C} \leq|y(r)|^{2} \leq C\left|y\left(r_{0}\right)\right|^{2} \quad\left(r \geq r_{0}\right)
$$

holds for every solution $y$ of $(*)$.

Proof. By Proposition 2 every fundamental system of $(*)$ is bounded on $\left[r_{0}, \infty\right)$. Consequently, there is $C>0$ such that

$$
|y(r)|^{2} \leq C\left|y\left(r_{0}\right)\right|^{2} \quad\left(r \geq r_{0}\right)
$$

holds for all solutions $y$ of $(*)$. Let $z$ be the solution of $(*)$ with initial value $z_{1}\left(r_{0}\right)=-y_{2}\left(r_{0}\right), z_{2}\left(r_{0}\right)=y_{1}\left(r_{0}\right)$; as the Wronskian is constant. we have

$$
\left|y\left(r_{0}\right)\right|^{4}=\left|\begin{array}{cc}
y_{1}\left(r_{0}\right) & -y_{2}\left(r_{0}\right) \\
y_{2}\left(r_{0}\right) & y_{1}\left(r_{0}\right)
\end{array}\right|^{2}=\left|\begin{array}{cc}
y_{1} & z_{1} \\
y_{2} & z_{2}
\end{array}\right|^{2} \leq|y|^{2}|z|^{2} \leq\left.\left. C|y|^{2}\right|_{z}\left(r_{0}\right)\right|^{2},
$$

and the assertion follows.

Remark 5. If we specialize $L \equiv 0, M \equiv 1$, Proposition 2 states that for all real $\lambda$, all solutions of the differential equation

$$
\left(\sigma_{2} p+\sigma_{3}+q I_{2}\right) u=\lambda u
$$

are bounded at infinity provided $\lim _{r \rightarrow \infty} q(r)=\infty$ and $1 /(q-\lambda) \in B V(\cdot, \infty)$ (which is equivalent to $1 / q \in B V(\cdot, \infty)$. cf. Remark 8 in the Appendix). These assumptions are equivalent to the hypotheses of Theorem 1 of [12]. since a bounded function of locally bounded variation has bounded total variation if and 
only if it has bounded positive variation.

Remark 6. Conversely, seeing that, in contrast to the rather large mass term $\sigma_{3} m$, the angular momentum term $(k / r) \sigma_{1}$ in the eigenvalue equation for $h_{k}$ is a smooth function on $(0, \infty)$ decaying at infinity, one may at first be tempted to treat it as a perturbation of the equation without angular momentum term, which can be handled by a simpler version of Proposition 2 (this line of attack was sketched in [12] Remark 2, for the case $m=1$ ). However, the unitary transformation used to turn the angular momentum term into an integrable perturbation ([11] Lemma 3), involves the derivatives of both the angular momentum and the mass (or scalar potential) term, leading to the stronger requirement

$$
\text { (A4) } \cdot \frac{m^{\prime}}{m^{2}} \in L^{1}(\cdot, \infty)
$$

instead of (A4) in Theorem 1. (For example, if $q(r)>c r^{\varepsilon}$ for some $c, \varepsilon>0$, and $m$ is periodic, then (A4) is satisfied, but (A4)' is not.) Therefore we prefer this strong version of Proposition 2, in which $L$ and $M$ enter in a perfectly symmetric way. On quite different grounds, Proposition 2 will also prove useful in Section 4.

The basic idea for the proof of Proposition 2 is essentially that developed in the proof of Theorem 1 of [12]. Instead of the pointwise norm $|u|:=$ $\sqrt{\left|u_{1}\right|^{2}+\left|u_{2}\right|^{2}}$ of the solution itself, we study the behaviour of an associated quantity $R(r)$, which may be interpreted as the major radius of the elliptical orbit in the $\left(u_{1}, u_{2}\right)$ plane on which the (real-valued) solution would be running if the coefficients were held constant at their value at $r$. In the case $L \equiv 0$ considered in [12] the major axes of the ellipse coincide with the $u_{1}{ }^{-}$, $u_{2}$-axes; in the general situation captured by Proposition 2, however, the ellipse is oblique, which renders the function $R$, as expressed in terms of the solution $u$, considerably more complicated. - Note that our $R$ corresponds to the $R^{2}$ of [12].

Proof of Proposition 2. Let $u \in A C_{\mathrm{loc}}([a, \infty))$ be a solution of (*) with real-valued components. By hypothesis, there is $r_{0}>0$ such that $Q(r)-W(r)>0$ $\left(r \geq r_{0}\right)$, and

$$
\frac{W}{Q-W}, \frac{M}{Q-W}, \frac{L}{Q-W} \in B V\left(\left[r_{0}, \infty\right)\right) .
$$

On $\left[r_{0}, \infty\right)$ we consider the function

$$
R:=\frac{1}{Q-W}\left(\left(u_{1}^{2}+u_{2}^{2}\right) Q+\left(u_{1}^{2}-u_{2}^{2}\right) M+2 u_{1} u_{2} L\right)
$$


Note that

$$
\frac{Q}{Q-W}=1+\frac{W}{Q-W} \in B V\left(\left[r_{0}, \infty\right)\right) .
$$

Thus we have $R \in B V_{\text {loc }}\left(\left[r_{0}, \infty\right)\right)$ by Proposition 3 in the Appendix.

For $t_{2} \geq t_{1} \geq r_{0}$, the formula for integration by parts for Stieltjes integrals (Fichtenholz [5] §577) and the local absolute continuity of $u$ yield:

$$
\begin{aligned}
R\left(t_{2}\right)-R\left(t_{1}\right)=\int_{t_{1}}^{t_{2}}\left(\left(u_{1}^{2}+u_{2}^{2}\right)^{\prime} Q+\left(u_{1}^{2}-u_{2}^{2}\right)^{\prime} M+\left(2 u_{1} u_{2}\right)^{\prime} L\right) \frac{1}{Q-W} \\
+\int_{t_{1}}^{t_{2}}\left(u_{1}^{2}+u_{2}^{2}\right) d\left(\frac{W}{Q-W}\right)+\int_{t_{1}}^{t_{2}}\left(u_{1}^{2}-u_{2}^{2}\right) d\left(\frac{M}{Q-W}\right) \\
+\int_{t_{1}}^{t_{2}} 2 u_{1} u_{2} d\left(\frac{L}{Q-W}\right) .
\end{aligned}
$$

As $u$ is a solution of $(*)$ and hence

$$
u_{1}^{\prime}=-L u_{1}-(Q-M) u_{2}, \quad u_{2}^{\prime}=(Q+M) u_{1}+L u_{2},
$$

a straightforward calculation shows that the integrand of the first integral vanishes identically. Estimating $\left|u_{1}^{2}-u_{2}^{2}\right|, 2\left|u_{1} u_{2}\right| \leq|u|^{2}$, and

$$
R=u_{1}^{2}+u_{2}^{2}+\frac{1}{Q-W}\left(\sqrt{W+M} u_{1}+(\operatorname{sgn} L) \sqrt{W-M} u_{2}\right)^{2} \geq|u|^{2} .
$$

and noting that $\underset{\left[t_{1}, t_{2}\right]}{\operatorname{Var}} f=\int_{t_{1}}^{t_{2}}|d f|$ holds for every function $f$ of locally bounded variation, we find

$$
R\left(t_{2}\right)-R\left(t_{1}\right) \leq\left(\operatorname{Var}_{\left[t_{1}, t_{2}\right]} \frac{W}{Q-W^{7}}+\operatorname{Var}_{\left[t_{1}, t_{2}\right]} \frac{M}{Q-W}+\operatorname{Var}_{\left[t_{1}, t_{2}\right]} \frac{L}{Q-W}\right) \sup _{\left[t_{1} t_{2}\right]} R .
$$

By Lemma 2 of [12], it follows that $R$, and consequently $u$, is bounded in $\left[r_{0}, \infty\right)$. This concludes the proof in the general case.

In the case $M \equiv 0$, we consider the function

$$
R:=u_{1}^{2}+u_{2}^{2}+\frac{L}{Q-L}\left(u_{1}+u_{2}\right)^{2} .
$$

By virtue of (C2) and (C3)' there is some $\varepsilon \in(0,1)$ and $r_{0}>0$ such that $|L(r)|$ $\leq \varepsilon Q(r) \quad\left(r \geq r_{0}\right)$, and $L /(Q-L) \in B V\left(\left[r_{0}, \infty\right)\right)$. Therefore $R \in B V_{\text {loc }}\left(\left[r_{0}, \infty\right)\right)$, and it is not difficult to check that

$$
\frac{2 L(r)}{Q(r)-L(r)} \geq \frac{-2 \varepsilon}{1+\varepsilon}>-1
$$

and hence $R(r) \geq \frac{1-\varepsilon}{1+\varepsilon}|u(r)|^{2}\left(r \geq r_{0}\right)$. By essentially the same calculation as above we infer that for $t_{2} \geq t_{1} \geq r_{0}$. 


$$
R\left(t_{2}\right)-R\left(t_{1}\right) \leq 2 \frac{1+\varepsilon}{1-\varepsilon} \sup _{\left[t_{1}, t_{2}\right]} R \cdot \operatorname{Var}_{\left[t_{1}, t_{2}\right]} \frac{L}{Q-L}
$$

and the assertion again follows by Lemma 2 of [12].

For $L \equiv 0$, take

$$
R:=u_{1}^{2}+u_{2}^{2}+\frac{2 M}{Q-M} u_{1}^{2}
$$

and proceed as above.

Now we conclude the proof of Theorem 1 , showing that under hypotheses $(\mathrm{A} 1)-(\mathrm{A} 4), Q:=q-\lambda, M:=m$ and $L(r):=k / r(r>a:=0)$ satisfy (C1) $-(\mathrm{C} 3)$, for every $\lambda \in \mathbb{R}$ and $k \in \mathbb{Z} \backslash\{0\}$.

(C1) and (C2) are obvious from (A1) and (A2), respectively. As in Proposition 2, abbreviate $W:=\sqrt{M^{2}+L^{2}}$. We first prove $W /(Q-W) \in$ $B V(\cdot, \infty)$, which is equivalent to $W / Q \in B V\left({ }^{\circ}, \infty\right)$ by Proposition 4 in the Appendix. By Proposition $3, W / Q=(W / M) \cdot(M / Q) \in B V(\cdot, \infty)$, since $M / Q \in$ $B V(\cdot, \infty)$ by $(\mathrm{A} 3), W / M \in L^{\infty}(\cdot, \infty) \cap A C_{\mathrm{loc}}(\cdot, \infty)$, and

$$
\frac{M}{Q} \cdot\left(\frac{W}{M}\right)^{\prime}=\frac{L\left(M L^{\prime}-L M^{\prime}\right)}{Q M W}=-\frac{k^{2} q}{r W Q}\left(\frac{m^{\prime}}{q m r}+\frac{1}{q r^{2}}\right) \in L^{1}(\cdot, \infty) .
$$

Similarly, we have $M /(Q-W)=(M / W) \cdot(W /(Q-W)) \in B V(\cdot, \infty)$. since $M / W \in L^{\infty}(\cdot, \infty) \cap A C_{\mathrm{loc}}(\cdot, \infty)$, and

$$
\frac{W}{Q-W} \cdot\left(\frac{M}{W}\right)^{\prime}=\frac{M Q}{W(Q-W)} \cdot \frac{L\left(M^{\prime} L-M L^{\prime}\right)}{Q M W} \in L^{1}(\cdot \infty)
$$

Finally, $L /(Q-W)=(L / M) \cdot(M /(Q-W)) \in B V(\cdot, \infty)$, since $L / M \in L^{\infty}(\cdot, \infty) \cap$ $A C_{\text {loc }}(\cdot, \infty)$, and

$$
\frac{M}{Q-W} \cdot\left(\frac{L}{M}\right)^{\prime}=-\frac{k}{r^{2}(Q-W)}-\frac{k q}{Q-W} \cdot \frac{m^{\prime}}{q m r} \in L^{1}(\cdot, \infty) .
$$

\section{\$. The Case $m \equiv q$}

In this section we prove Theorem 2. Since it is shown in [20] that $\sigma(H) \cap$ $(0, \infty)$ is purely discrete, and since the spherically symmetric operator $H$ admits separation in spherical polar coordinates, it is sufficient to prove that, for each non-zero integer $k .(-\infty, 0) \subset \sigma_{a c}\left(h_{k}\right)$, and $\sigma_{s}\left(h_{k}\right) \cap(-\infty, 0)=\emptyset$.

We shall make use of the Gilbert-Pearson theory (Gilbert and Pearson [6], Gilbert [7]. Behncke [1]), showing that for negative $\lambda$. the differential equation

$$
\left(\sigma_{2} p+q(r) \sigma_{3}+q(r) I_{2}+\frac{k}{r} \sigma_{1}\right) u=\lambda u
$$


does not possess a subordinate solution at $\infty$, i.e. that any two non-trivial solutions $v$ and $w$ of $(* *), \lambda<0$, satisfy

$$
\liminf _{r \rightarrow \infty} \frac{\int_{r_{0}}^{r}|v|^{2}}{\int_{r_{0}}^{r}|w|^{2}}>0
$$

for some $r_{0}>0$. We have to go back to this more general definition, bacause unlike the situation of Section 3, the solutions will be unbounded, as suggested by the asymptotics given in Remark 3 .

By a transformation which takes into account this expected growth, or decay, behaviour of the solutions, we obtain a differential equation which is again of Dirac type, with coefficients which (with the help of Lemma 1 below) can be shown to satisfy the hypotheses of Proposition 2. As in Section 2, we can therefore conclude that all solutions of the transformed equation are globally bounded at infinity; indeed Corollary 3 shows that all solutions of the transformed equation are of the same size. This does not immediately imply that the original equation has no subordinate solutions, since the transformation is unbounded; yet considering the oscillatory behaviour of the solutions, it turns out that the growing and decaying components are sufficiently well distributed between solutions to prevent the existence of a subordinate solution.

Lemma 1. Under the hypotheses of Theorem 2, let $\lambda \in \mathbb{R}$ and set $\gamma:=2 q-\lambda$. Then $r \in A C_{\mathrm{loc}}([0, \infty))$, and

$$
\frac{\gamma^{\prime}}{\gamma^{3 / 2}} \in B V(\cdot, \infty), \quad \frac{\gamma^{\prime}}{r \gamma^{3 / 2}} \in L^{1}(\cdot, \infty), \quad \lim _{r \rightarrow \infty} \frac{\gamma^{\prime}(r)}{\gamma(r)^{3 / 2}}=0
$$

Proof. There is $r_{0}>0$ such that $\gamma(r) \geq q(r) \geq 1\left(r \geq r_{0}\right)$, and $g:=2 q^{\prime} q^{-3 / 2} \in$ $B V\left(\left[r_{0}, \infty\right)\right) \cap L^{2}\left(\left[r_{0}, \infty\right)\right)$.

Applying Proposition 3 in the Appendix with $f:=(q / \gamma)^{3 / 2}$ (noting that $|f| \leq 1$,

$$
\left.\left|f^{\prime} g\right|=\left|\frac{3 \lambda q^{\prime 2}}{q r^{5 / 2}}\right| \leq 3|\lambda| \frac{q^{\prime 2}}{q^{7 / 2}} \in L^{1}\left(\left[r_{0}, \infty\right)\right)\right) \text {, }
$$

we obtain $\gamma^{\prime} \gamma^{-3 / 2}=f g \in B V\left(\left[r_{0}, \infty\right)\right)$.

Furthermore, $\left|\gamma^{\prime} \gamma^{-3 / 2}\right| \leq|g|$, and thus the second assertion follows by the Schwarz inequality. Finally, the last assertion follows the fact that $g$, as a square integrable function of bounded variation on $\left[r_{0}, \infty\right)$, must converge to 0 at infinity.

Proof of Theorem 2. Let $\lambda<0, k \in \mathbb{Z} \backslash\{0\}$, and $u, y$ nontrivial solutions of $(* *)$ with real-valued components. On a right half-axis on which $\gamma:=2 q-\lambda$ is 
positive, we consider the functions

$$
v:=\left(\begin{array}{c}
(\gamma / \Lambda)^{1 / 4} u_{1} \\
(\Lambda / \gamma)^{1 / 4} u_{2}
\end{array}\right), \quad w:=\left(\begin{array}{c}
(\gamma / \Lambda)^{1 / 4} y_{1} \\
(\Lambda / \gamma)^{1 / 4} y_{2}
\end{array}\right) \quad(\text { with } \Lambda:=|\lambda|)
$$

then $v$ and $w$ are solutions of the differential equation of Dirac type

$$
\left(\sigma_{2} p+L \sigma_{1}+Q I_{2}\right) v=0,
$$

where $L=(k / r)-\left(\gamma^{\prime} / 4 r\right), Q=\sqrt{\Lambda \gamma}$.

We observe that $\lim _{r \rightarrow \infty} Q(r)=\infty$, and

$$
\frac{L(r)}{Q(r)}=\frac{k}{r \sqrt{\Lambda \gamma}}-\frac{r^{\prime}(r)}{4 \sqrt{\Lambda} r^{(r)^{3 / 2}}} \rightarrow 0 \quad(r \rightarrow \infty) ;
$$

moreover, $L / Q \in B V(\circ, \infty)$ as a result of Lemma 1 , since

$$
\left(\frac{1}{r \sqrt{\gamma}}\right)^{\prime}=-\frac{\gamma^{\prime}}{2 r \gamma^{3 / 2}}-\frac{1}{r^{2} \sqrt{\gamma}} \in L^{1}(\cdot, \infty) .
$$

In particular, by Proposition 4 in the Appendix, there is $r_{0}>0$ such that $2|L(r)| \leq Q(r) \quad\left(r \geq r_{0}\right)$ and $L /(Q-L) \in B V\left(\left[r_{0}, \infty\right)\right)$. Therefore $L$ and $Q$ satisfy conditions (C1), (C2) and (C3)' of Proposition 2 with $M \equiv 0$.

Corollary 3 shows that there is a constant $C>0$ such that

$$
\frac{\left|v\left(r_{0}\right)\right|^{2}}{C} \leq|v(r)|^{2} \leq C\left|v\left(r_{0}\right)\right|^{2}, \quad \frac{\left|w\left(r_{0}\right)\right|^{2}}{C} \leq|w(r)|^{2} \leq C\left|w\left(r_{0}\right)\right|^{2}
$$

i.e. that $v$ and $w$ are of the same size: we now use this estimate to prove that

$$
\liminf _{r \rightarrow \infty} \frac{\int_{r_{0}}^{r}\left(u_{1}^{2}+u_{2}^{2}\right)}{\int_{r_{0}}^{r}\left(y_{1}^{2}+y_{2}^{2}\right)}>0 .
$$

To this end, we study the oscillation behaviour of $v$ by means of the Prüfer transformation.

There are locally absolutely continuous functions $\rho:\left[r_{0}, \infty\right) \rightarrow(0, \infty)$, and $\vartheta:\left[r_{0}, \infty\right) \rightarrow \mathbb{R}$, such that $\vartheta\left(r_{0}\right) \in[-3 \pi / 4,5 \pi / 4)$, and

$$
v=\varrho\left(\begin{array}{c}
\cos \vartheta \\
\sin \vartheta
\end{array}\right)
$$

$\vartheta$ is a solution of the differential equation

$$
\vartheta^{\prime}(r)=L(r) \sin 2 \vartheta(r)+Q(r) .
$$

Introducing a new independent variable by the transformation $s(r):=\int_{r_{0}}^{r} Q$, and setting $\Theta(s(r))=\vartheta(r)$, we have 


$$
\Theta^{\prime}(s(r))=1+\frac{L(r)}{Q(r)} \sin 2 \Theta(s(r)) .
$$

From the definition of $\gamma_{0}$, we find that $\Theta^{\prime}(s) \in[1 / 2,3 / 2] \quad(s \geq 0)$.

For $n \in \mathbb{N}$ we define

$$
\begin{aligned}
& J_{n}:=\{s \geq 0 \mid \Theta(s) \in[-3 \pi / 4,-\pi / 4]+n \pi\}, \\
& K_{n}:=\{s \geq 0 \mid \Theta(s) \in[-\pi / 4, \pi / 4]+n \pi\} ;
\end{aligned}
$$

then $\frac{\pi}{3} \leq\left|J_{n}\right|,\left|K_{n}\right| \leq \pi \quad\left(n \in \mathbb{N}_{0}\right)$

$\left(\mid \cdot\right.$ |here denotes the length of the interval). Adjusting $r_{0}$ if necessary, we can assume that $0=\inf J_{1}$.

Observing that $\gamma>\Lambda$, and $Q=\sqrt{r \Lambda}$, we find for $r \geq r_{0}$

$$
\begin{aligned}
\frac{\int_{r_{0}}^{r}\left(u_{1}^{2}+u_{2}^{2}\right)}{\int_{r_{0}}^{r}\left(y_{1}^{2}+y_{2}^{2}\right)} & =\frac{\int_{r_{0}}^{r}\left(\sqrt{\Lambda / \gamma} v_{1}^{2}+\sqrt{\gamma / \Lambda} v_{2}^{2}\right)}{\int_{r_{0}}^{r}\left(\sqrt{\Lambda / \gamma} w_{1}^{2}+\sqrt{r / \Lambda} w_{2}^{2}\right)} \geq \frac{\int_{r_{0}}^{r} \sqrt{\gamma / \Lambda} v_{2}^{2}}{\int_{r_{0}}^{r} \sqrt{r / \Lambda}\left(w_{1}^{2}+w_{2}^{2}\right)} \\
& =\frac{\int_{0}^{s} V_{2}^{2}}{\int_{0}^{s}\left(W_{1}^{2}+W_{2}^{2}\right)} \geq \frac{\int_{0}^{s} V_{2}^{2}}{C\left|w\left(r_{0}\right)\right|^{2} s(r)}
\end{aligned}
$$

where $v(r)=V(s(r)), w(r)=W(s(r))$ and $C$ is the constant from Corollary 3 . Now setting $N(r):=\max \left\{n \in \mathbb{N} \mid K_{n} \subset[0, s(r)]\right\}$ and noting that on $J_{n}$,

$$
V_{2}^{2} \geq \frac{V_{1}^{2}+V_{2}^{2}}{2} \geq \frac{\left|v\left(r_{0}\right)\right|^{2}}{2 C}
$$

we conclude that

$$
\begin{aligned}
\frac{\int_{r_{0}}^{r}\left(u_{1}^{2}+u_{2}^{2}\right)}{\int_{r_{0}}^{r}\left(y_{1}^{2}+y_{2}^{2}\right)} \geq \frac{\sum_{n=1}^{N(r)} \int_{J_{n}} V_{2}^{2}}{C\left|w\left(r_{0}\right)\right|^{2} s(r)} \geq \frac{\pi\left|v\left(r_{0}\right)\right|^{2} N(r)}{6 C^{2}\left|w\left(r_{0}\right)\right|^{2} \sum_{n=1}^{N(r)+1}\left(\left|J_{n}\right|+\left|K_{n}\right|\right)} \\
\geq \frac{\left|v\left(r_{0}\right)\right|^{2}}{12 C^{2}\left|w\left(r_{0}\right)\right|^{2}} \cdot \frac{N(r)}{N(r)+1} \rightarrow \frac{\left|v\left(r_{0}\right)\right|^{2}}{12 C^{2}\left|w\left(r_{0}\right)\right|^{2}}>0 \quad(r \rightarrow \infty)
\end{aligned}
$$

\section{Appendix}

The great advantage of regularity assumptions on coefficient functions in terms of their differentiability is based on the existence of a linear and multiplicative differential calculus, by which subsequent estimates of terms containing these coefficient functions are conveniently accessible. Unfortunately, 
such a calculus does not exist for the variation of functions on the real line, which generally behaves like the integral of the absolute value of the derivative of the function, but cannot be treated as such unless the function is differentiable or at least locally absolutely continuous. The approximation of functions of bounded variation by differentiable functions, as in Weidmann [16] p. 368, is of limited scope (and can hardly be effected in conditions like our (B2)), whereas going back to the definition of the variation,

$$
\operatorname{Var}_{I} f:=\sup \sum_{j=1}^{N}\left|f\left(x_{j}\right)-f\left(x_{j-1}\right)\right|
$$

(where the supremum ranges over all finite partitions $a=x_{0}<x_{1}<\ldots<x_{N}=b$, $N \in \mathbb{N}$, of the interval $I=[a, b])$, is tedious and tends to obscure the line of reasoning. On the other hand, it appears unsatisfactory to assume differentiability of coefficients in situations where only their variation, but not their derivatives occur naturally. Therefore we have collected in this appendix some properties of functions of bounded variation which have served as a substitute for the differential calculus in the main body of this paper, so that any approximation or reference to the definition above could be avoided there, while working with minimal regularity requirements. In particular, Proposition 3 is interesting as a weaker surrogate for the product rule, and is frequently used in both Sections 2 and 3. Similarly. Proposition 4 replaces the quotient rule in several instances in our proofs. The outlook of Proposition 5 is more restricted to the purpose of our paper; it shows that condition (A3) of Theorem 1 is valid for all $\lambda \in \mathbb{R}$ if it holds for two distinct values of $\lambda$.

Proposition 3. Let $I \subset \mathbb{R}$ be an interval and $g \in B V(I), f \in A C_{\mathrm{loc}}(I) \cap$ $L^{\infty}(I)$. Then

$$
\underset{I}{\operatorname{Var}} f g \leq \int_{I}\left|f^{\prime} g\right|+\|f\|_{\infty} \underset{I}{\operatorname{Var}} g:
$$

in particular, $f^{\prime} g \in L^{1}(I)$ implies $f g \in B V(I)$.

Proof. Let $x, y \in I, x<y$. By the Jordan decomposition theorem (Fichtenholz [5] §570), there are non-decreasing functions $g_{+}, g_{-}: I \rightarrow \mathbb{R}$ such that $g=g_{+}-g_{-}$, and $\operatorname{Var}_{[x, y]} g=g_{+}(y)+g_{-}(y)-g_{+}(x)-g_{-}(x)$. The formula for integration by parts for Stieltjes incegrals gives

$$
f(y) g(y)-f(x) g(x)=\int_{x}^{y} f^{\prime} g+\int_{x}^{y} f d g_{+}-\int_{x}^{y} f d g_{-} .
$$

The mean value theorem for Stieltjes integrals (Fichtenholz [5] §582) implies that there exist $z_{+}, z_{-} \in[x, y]$ such that 


$$
\int_{x}^{y} f d g_{ \pm}=f\left(z_{ \pm}\right)\left(g_{ \pm}(y)-g_{ \pm}(x)\right)
$$

thus

$$
|f(y) g(y)-f(x) g(x)| \leq \int_{x}^{y}\left|f^{\prime} g\right|+\|f\|_{\infty}\left(g_{+}(y)-g_{+}(x)\right)+\|f\|_{\infty}\left(g_{-}(y)-g_{-}(x)\right) .
$$

Applying this estimate to the individual intervals of partitions of $I$, we obtain the assertion.

Remark 7. The simpler estimate $\operatorname{Var}_{I} f g \leq\|f\|_{\infty} \operatorname{Var}_{I} g+\|g\|_{\infty} \operatorname{Var}_{I} f$ (for $f, g \in B V(I)$ ) is tempting; but using this instead of Proposition 3 in the final step of the proof of Theorem 1 requires the slightly stronger condition (A4)' (cf. Remark 6) instead of (A4).

Proposition 4. Let $I \subset \mathbb{R}$ be an interval and $f, g: I \rightarrow \mathbb{R}$. Assume $g>0$ and $\varepsilon:=\sup _{I}|f| / g<1$. Then

$$
(1-\varepsilon)^{2} \operatorname{Var}_{I} \frac{f}{g-f} \leq \operatorname{Var}_{I} \frac{f}{g} \leq(1+\varepsilon)^{2} \operatorname{Var}_{I} \frac{f}{g-f}
$$

in particular, $f / g \in B V(I)$ if and only if $f /(g-f) \in B V(I)$.

Proof. For $x, y \in I$ we have

$$
\left|\frac{f(y)}{g(y)-f(y)}-\frac{f(x)}{g(x)-f(x)}\right|=\frac{g(x)}{g(x)-f(x)} \cdot \frac{g(y)}{g(y)-f(y)} \cdot\left|\frac{f(y)}{g(y)}-\frac{f(x)}{g(x)}\right| .
$$

and the assertion follows observing that

$$
\frac{1}{1+\varepsilon} \leq \frac{g}{g-f} \leq \frac{1}{1-\varepsilon}
$$

Proposition 5. Let $a>0, m, q:(a, \infty) \rightarrow \mathbb{R}$ functions such that

$$
\lim _{r \rightarrow \infty} q(r)=\infty, \quad \limsup _{r \rightarrow \infty} \frac{|m(r)|}{q(r)}<1 .
$$

Then $\left\{\lambda \in \mathbb{R} \mid \frac{m}{q-\lambda} \in B V(\cdot, \infty)\right\}$ is either empty. or the whole real line, or has exactly one element.

Proof. Assume this set has two distinct elements $\lambda_{1}, \lambda_{2}$ : we then show that it is all of $\mathbb{R}$. Let $\lambda \in \mathbb{R}$. Then there is $r_{0}>0$ such that $m /\left(q-\lambda_{\jmath}\right) \in B V\left[r_{0}, \infty\right)$. and

$$
q(r)-\lambda \geq \frac{q(r)-\lambda_{1}}{2} \geq 1 \quad\left(r \geq r_{0}, j \in\{1,2\}\right)
$$


Defining $\mu_{j}:=\frac{\lambda_{j}-\lambda}{\lambda_{2}-\lambda_{1}}(j \in\{1,2\})$, and noting that $1=\mu_{2}-\mu_{1}, \lambda=\lambda_{1} \mu_{2}-\lambda_{2} \mu_{1}$, we find

$$
\begin{aligned}
& \left|\frac{m(y)}{q(y)-\lambda}-\frac{m(x)}{q(x)-\lambda \mid}\right|=\frac{|m(y) q(x)-\lambda m(y)-m(x) q(x)-\lambda m(x)|}{(q(y)-\lambda)(q(x)-\lambda)} \\
& \quad \leq 4\left|\mu_{2}\right|\left|\frac{m(y)}{q(y)-\lambda_{1}}-\frac{m(x)}{q(x)-\lambda_{1}}\right|+4\left|\mu_{1}\right|\left|\frac{m(y)}{q(y)-\lambda_{2}}-\frac{m(x)}{q(x)-\lambda_{2}}\right|
\end{aligned}
$$

for $x, y \in\left[r_{0}, \infty\right)$. Thus we obtain

$$
\underset{\left[r_{0, \infty)}\right.}{\operatorname{Var}} \frac{m}{q-\lambda} \leq 4\left|\mu_{2}\right| \underset{\left[r_{0}, \infty\right)}{\operatorname{Var}} \frac{m}{q-\lambda_{1}}+4\left|\mu_{1}\right| \underset{\left[r_{0}, \infty\right)}{\operatorname{Var}} \frac{m}{q-\lambda_{2}}<\infty
$$

Remark 8. If $m$ is constant, the $\lambda m$ terms in the numerator cancel, and it is easy to see that $m /(q-\lambda)$ is of bounded variation either for every, or for no real value of $\lambda$ (as observed in [12]).

\section{Ackmowledgement}

The greater part of this work has been done during the visit of the second author at Munich University. He would like to express his gratitude to Professors H. Kalf, E. Wienholtz and A. M, Hinz for their hospitality.

\section{References}

[1] Behncke, H., Absolute continuity of Hamiltonians with von Neumann Wigner potentials. Proc. Amer. Math. Soc., 111 (1991), 373-384.

[2] Dunford, N. and Schwartz, J. T., Linear operators II, Wiley, New York, 1963.

[3] Erdélyi, A., Note on a paper by Titchmarsh. Quart. J. Math. Oxford (2), 14 (1963), 147-152.

[4] Evans. W. D. and Harris, B. J., Bounds for the point spectra of separated Dirac operators. Proc. Roy. Soc. Edinburgh, 88A (1981), 1-15.

[5] Fichtenholz, G. M., Differential-und Integralrechnung III, Deutscher Verlag der Wissenschaften, Berlin, 1973.

[6] Gilbert, D. J. and Pearson. D. B., On subordinacy and analysıs of the spectrum of one-dimensional Schrodinger operators, J. Math. Anal. Appl., 128 (1987), 30-56.

[7] Gilbert. D. J., On subordinacy and analysis of the spectrum of Schrödinger operators with two singular endpoints, Proc. Roy. Soc. Edinburgh, 112A (1989), 213-229.

[8] Hinton, D. B. and Shaw, J. K., Dirac systems with discrete spectra. Can. J. Math., 39 (1987), $100-122$.

[9] Roos, B. W. and Sangren. W. C., Spectra for a pair of singular first order differential equations, Proc. Amer. Math. Soc., 12 (1961), 468-476.

[10] Rose, M. E. and Newton. R. R., Properties of Dirac wave functions in a central field. Phys. Rev. (2), 82 (1951), 470-477.

[11] Schmidt. K. M., Dense point spectrum and absolutely continuous spectrum in spherically symmetric Dirac operators, Fontm Math.. 7 (1995), 459-475. 
[12] - Absolutely continuous spectrum of Dirac systems with potentials infinite at infinity, Math. Proc. Camb. Phrl. Soc., 122 (1997), 377-384.

[13] Simon, B., Bounded eigenfunctions and absolutely continuous spectra for one-dimensional Schrödinger operators, Proc. Amer. Math. Soc., 124 (1996). 3361-3369.

[14] Thaller, B., The Dirac equation, Springer, Berlin, 1992.

[15] Titchmarsh, E. C., On the nature of the spectrum in problems of relativistic quantum mechanics, Quart. J. Math. Oxford (2), 12 (1961), 227-240.

[16] Weidmann, J., Oszillationsmethoden für Systeme gewöhnlicher Differential gleichungen, Math. Z., 119 (1971), 349-373.

[17] - Absolutstetiges Spektrum bei Sturm-Liouville-Operatoren und Diracsystemen, Math. Z., 180 (1982), 423-427.

[18] - Spectral theory of ordinary differential operators, Lect. Notcs Math. 1258. Springer, Berlin, 1987.

[19] - Uniform nonsubordinacy and the absolutely continuous spectrum, Analysis, 16 (1996), 89-99.

[20] Yamada, O., On the spectrum of Dirac operators with the unbounded potential at infinity. Hokkaido Math. J., 26 (1997). 439-449. 
